Supplementary Information for

\title{
Effect of Solvent Quality on the Phase Behavior of Polyelectrolyte Complexes
}

Lu Li, ${ }^{1}$ Artem M. Rumyantsev, ${ }^{1}$ Samanvaya Srivastava,, ,* Siqi Meng, ${ }^{1}$ Juan J. de Pablo1,3,* and Matthew V. Tirrell1,3,*

1Pritzker School of Molecular Engineering, The University of Chicago, Chicago, IL 60637, USA

${ }^{2}$ Chemical and Biomolecular Engineering, University of California, Los Angeles, Los Angeles, CA 90095, USA

${ }^{3}$ Center for Molecular Engineering and Materials Science Division, Argonne National Laboratory, Lemont, IL 60439, USA

Corresponding Authors

S. S.: samsri@ucla.edu

J. J. d. P.: depablo.uchicago.edu

M. V.T.: mtirrell@uchicago.edu

Table of Contents

Supplementary Figures S1-S2 


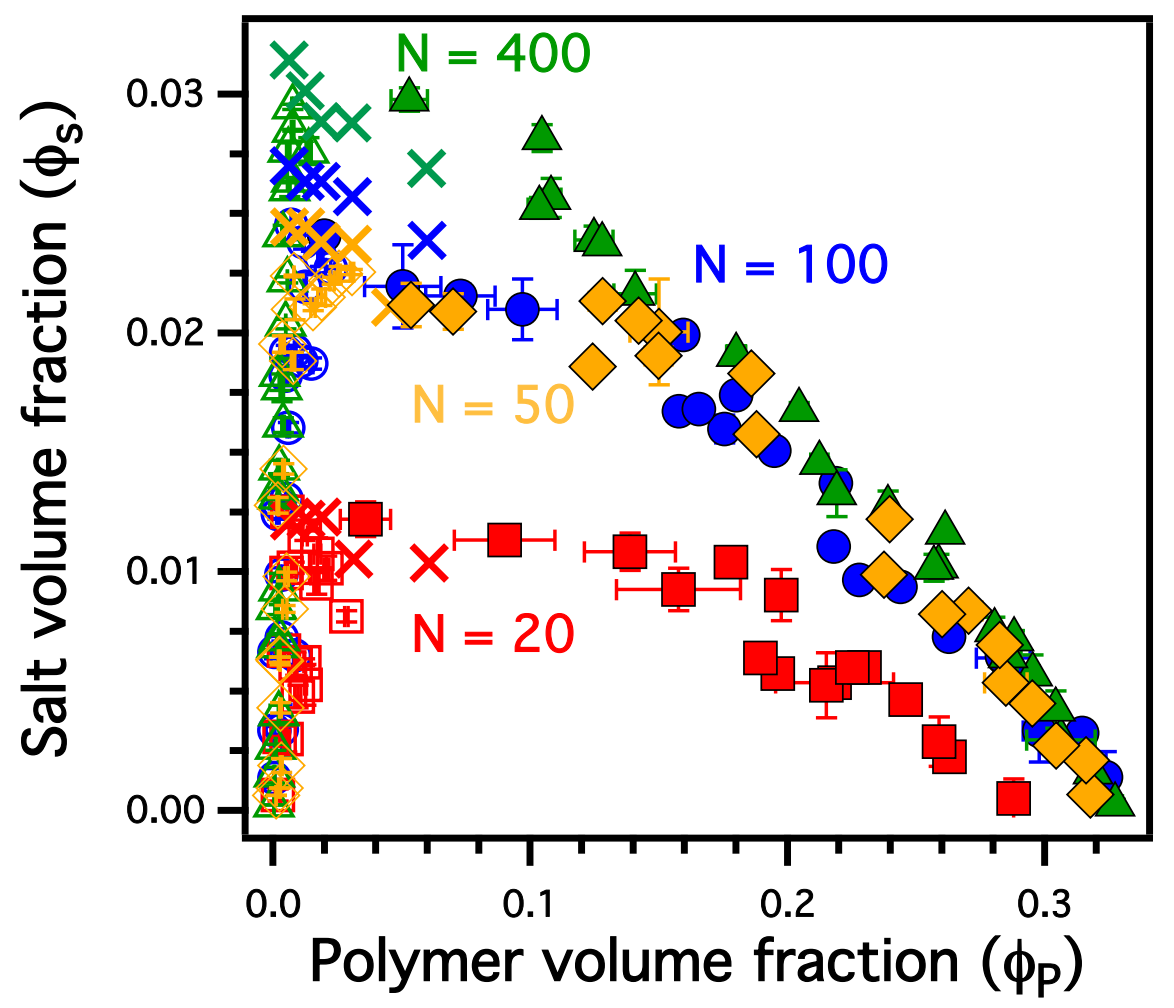

Figure S1. Experimentally determined phase diagrams of PLK-PRE system. The solid (complex phase) and open (supernatant phase) red squares, yellow diamonds, blue circles, and green triangles were the results obtained from TGA measurements for PLK-PRE systems with chain length of $20,50,100$, and 400 repeat units, respectively. The red, yellow, blue, and green crosses were the results converted from calculation based on salt resistance concentration measurements. Reprinted (adapted) with permission from L. Li et al., Macromolecules 51,8 (2018). Copyright (2018) American Chemical Society. 

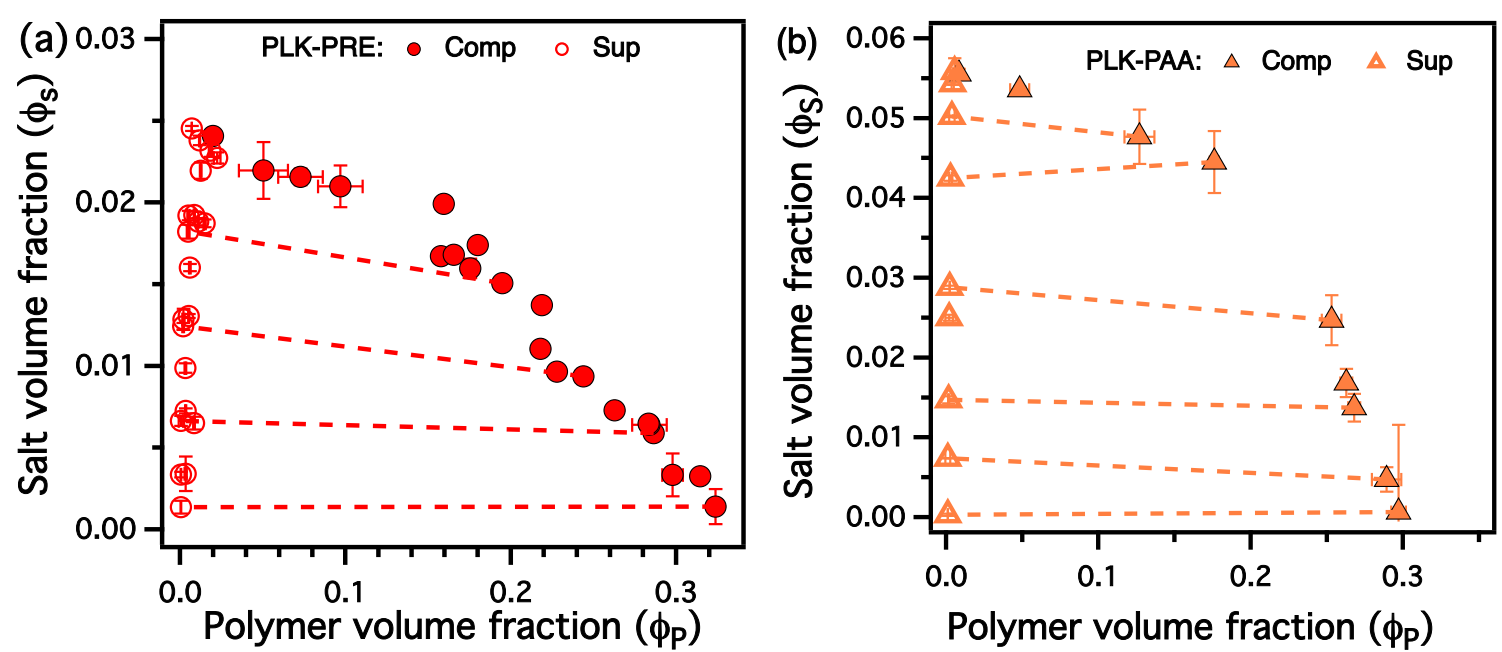

Polymer volume fraction $\left(\phi_{\mathrm{P}}\right)$
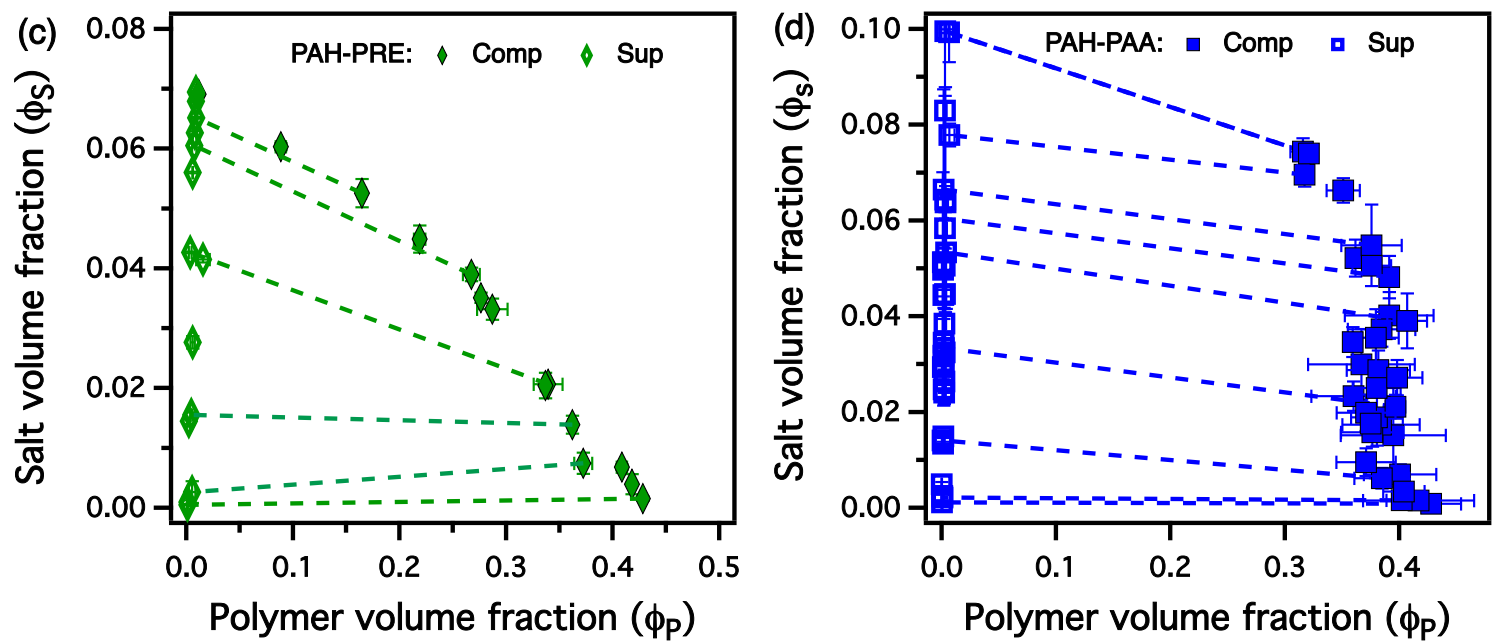

Figure S2. Individual phase diagrams of PLK-PRE (red), PLK-PAA (orange), PAH-PRE (green), and PAH-PAA (blue) systems. Selective tie-lines were drawn connecting complex phases (solid symbols) and corresponding supernatant phases (open symbols). Data shown on the both branches of the phase diagrams were obtained from thermogravimetric analysis measurements at different total polymer concentrations and added salt concentrations. Every data point was calculated based on at least three measurements. The standard deviation in the measurements are shown as error bars associated with each data point. 